\title{
Se former pour éduquer : la spatialité comme ressource(s). Exercices de pensée critique géographique d'étudiantes et d'étudiants
}

\section{Teacher training: Spatiality as a resource(s). Student exercises in geographical critical thinking Formarse para educar: la espacialidad como recurso(s). Ejercicios de pensamiento crítico geográfico de alumnos y alumnas}

\section{Anne-Laure Le Guern}

Volume 47, numéro 2, automne 2019

Éduquer aux sciences humaines et sociales

URI : https://id.erudit.org/iderudit/1066448ar

DOI : https://doi.org/10.7202/1066448ar

Aller au sommaire du numéro

Éditeur(s)

Association canadienne d'éducation de langue française

ISSN

1916-8659 (numérique)

Découvrir la revue

Citer cet article

Le Guern, A.-L. (2019). Se former pour éduquer : la spatialité comme ressource(s). Exercices de pensée critique géographique d'étudiantes et d'étudiants. Éducation et francophonie, 47(2), 66-82.

https://doi.org/10.7202/1066448ar
Résumé de l'article

La question posée dans cet article est de savoir si les étudiantes et les étudiants qui se forment pour enseigner la discipline scolaire appelée, en France, « histoire-géographie » ont une appréhension particulière de leur futur métier d'enseignant en raison même de leur discipline de formation et d'exercice professionnel. Les savoirs disciplinaires leur servent-ils de ressources professionnelles? L'étude d'un corpus de portfolios de formation et d'évaluation d'étudiantes et d'étudiants se préparant à l'exercice du métier montre des usages différenciés de la spatialité. L'article expose une grille d'analyse qui permet d'identifier les mobilisations disciplinaires et les conditions d'un exercice de pensée réflexive et critique à fort enjeu éducatif.

Tous droits réservés $@$ Association canadienne d’éducation de langue française, Ce document est protégé par la loi sur le droit d’auteur. L’utilisation des 2019 services d'Érudit (y compris la reproduction) est assujettie à sa politique d'utilisation que vous pouvez consulter en ligne. 


\section{Se former pour éduquer : la spatialité comme ressource(s). Exercices de pensée critique géographique d'étudiantes et d'étudiants}

Anne-Laure LE GUERN

Université de Caen Normandie, France

\section{RÉSUMÉ}

La question posée dans cet article est de savoir si les étudiantes et les étudiants qui se forment pour enseigner la discipline scolaire appelée, en France, «histoire-géographie» ont une appréhension particulière de leur futur métier d'enseignant en raison même de leur discipline de formation et d'exercice professionnel. Les savoirs disciplinaires leur servent-ils de ressources professionnelles? L'étude d'un corpus de portfolios de formation et d'évaluation d'étudiantes et d'étudiants se préparant à l'exercice du métier montre des usages différenciés de la spatialité. L'article expose une grille d'analyse qui permet d'identifier les mobilisations disciplinaires et les conditions d'un exercice de pensée réflexive et critique à fort enjeu éducatif. 


\section{ABSTRACT}

\section{Teacher training: Spatiality as a resource(s). Student exercises in geographical critical thinking}

Anne-Laure LE GUERN, University of Caen Normandy, France

The question posed in this article is whether or not future teachers being trained to teach «history-geography» as the course is called in France, are apprehensive about their future profession as teachers due to the training they received in their discipline and professional practice. Do they use their knowledge of the subject as a professional resource? The study of a corpus of portfolios and evaluations of students preparing for the profession shows differentiated uses of spatiality. The article presents an analysis grid identifying subject-specific mobilizations and conditions for an exercise in reflexive and critical thought with a major educational slant.

\section{RESUMEN}

\section{Formarse para educar: la espacialidad como recurso(s). Ejercicios de pensamiento crítico geográfico de alumnos y alumnas}

Anne-Laure LE GUERN, Universidad de Caen Normandia, Francia

La cuestión formulada en este artículo es saber si los estudiantes que se forman para enseñar la disciplina escolar que en Francia se denomina historia-geografía, poseen una aprensión particular de su futuro oficio de maestro, precisamente debido a su disciplina de formación y ejercicio profesional. ¿Los conocimientos disciplinarios les sirven de recursos profesionales? El estudio de un conjunto de portafolios de formación y evaluación de los estudiantes que se preparan para ejercer su oficio, muestra usos diferenciados de la espacialidad. El artículo expone una matriz de análisis que permite identificar las movilizaciones disciplinarias y las condiciones de un ejercicio del pensamiento reflexivo y crítico con desafíos educativos importantes.

Les travaux de recherche sur l'entrée dans le métier d'enseignant interrogent rarement les variations disciplinaires: ou c'est une cohorte de la même discipline ou ce sont des individus à l'intérieur d'une discipline donnée qui font l'objet d'une enquête. La question pourtant peut être posée: entre-t-on dans le métier de la même façon quand on enseigne telle discipline plutôt que telle autre? Existe-t-il des univers disciplinaires professionnalisants très distincts? Nous considérons que les recherches 
développent une vision restreinte des relations entre disciplines et métier tendant à faire des disciplines des contenus d'enseignement et non pas des outils intellectuels à proprement parler, comme si les disciplines ne valaient que comme matière scolaire et non pas comme discipline intellectuelle. Il s'agit, au contraire, de penser que ces disciplines sont des univers de pensée et d'action pour celles et ceux qui en sont les spécialistes, étudiants en formation professionnelle ou enseignants en exercice. Constitutives de leur identité (Prost, 1998), elles orientent (ou pourraient orienter) la lecture et la configuration, par ces étudiantes et étudiants, des situations d'enseignement observées ou mises en œuvre. Les disciplines sont à concevoir ainsi, selon nous, comme un outillage intellectuel de saisie du réel professionnel (Le Guern et Thémines, 2012).

Dans cet article, notre analyse se porte particulièrement sur les façons dont des étudiants et étudiantes formés, en France, à enseigner l'histoire-géographie, appréhendent, dans sa dimension spatiale, le métier d'enseignant. Nous émettons l'hypothèse que cette dimension ne leur est pas indifférente, du fait de leur formation initiale ainsi que des contenus géographiques qu'ils ont à enseigner (Thémines, 2006). Il s'agit donc de saisir quelles conceptions de l'espace sont mises en œuvre lors de premières approches, chez ces étudiantes et étudiants, des pratiques d'enseignement de l'histoire-géographie.

Notre question est la suivante: alors que les étudiants qui se préparent au métier d'enseignant d'histoire-géographie préparent le concours et accomplissent leurs premiers stages la même année, en quoi leur orientation disciplinaire les rend-elle sensibles aux enjeux spatiaux des situations d'enseignement? En particulier, est-ce que la littéracie visuelle (iconographies, cartographies, écritures multimodale et tabulaire) qui caractérise cette discipline scolaire ainsi qu'au moins l'une des deux disciplines éponymes universitaires (la géographie), les conduit à faire ressortir ces enjeux dans l'analyse de leurs débuts professionnels? La spatialité (Lussault, 2010), en d'autres termes, est-elle une ressource pour penser son métier et exercer sa réflexivité?

C'est à partir d'un corpus de portfolios de formation que les réponses à ces questions sont élaborées.

\section{LA DIMENSION SPATIALE DES APPRENTISSAGES PROFESSIONNELS EN HISTOIRE-GÉOGRAPHIE: ÉLÉMENTS DE CADRAGE}

Notre recherche ne relève pas d'une démarche d'expérimentation guidée par les formateurs et les formatrices. Il ne s'agit pas non plus, pour nous, d'identifier ce qui pourrait être considéré comme une bonne pratique. L'intention est de valider ou non l'hypothèse émise à propos de la discipline de formation. 
Se former pour éduquer : la spatialité comme ressource(s).

Exercices de pensée critique géographique d'étudiantes et d'étudiants

Notre étude s'inscrit dans une perspective qui considère les disciplines comme des univers de pensée et d'action. Ces univers "outillent» les personnes qu'ils forment dans leur appréhension des élèves, des savoirs, du monde et, bien sûr, du travail qui est le leur - travail qui consiste à faire rencontrer ces entités. S'agissant d'étudiantes et d'étudiants qui se préparent au métier d'enseignante et d'enseignant d'histoire-géographie, nous cherchons à savoir quelle mise en forme de l'apprentissage professionnel initial produit la discipline histoire-géographie, en tant qu'elle est un univers linguistique et sémiotique. Écrivent-ils leur appréhension de leur monde professionnel comme d'autres, formés par d'autres disciplines?

Nous nous focalisons sur la dimension spatiale de cet apprentissage professionnel. Par dimension spatiale, nous entendons le rapport à l'espace et à la matérialité, non pas considérée en soi, mais pour les êtres humains ou des êtres non humains en tant qu'ils ont «à faire avec l'espace [...] qui leur est fourni par le contexte d'expérience dans lequel ils sont insérés, au gré des circonstances qui sont les leurs» (Lussault, 2010, p. 12). Pour éviter une méprise au sujet de ce que nous entendons par matérialité, ajoutons que nous retenons la leçon de la linguistique saussurienne: le signifié (Sé) est bien ce qui est visé, mais toujours à partir d'une matérialité, celle du signifiant, qu'il soit donné à voir ou à entendre. On pourrait ajouter aussi à sentir, d'une manière générale, que ce soit par l'odorat ou le toucher. Mais ce signifiant est bien souvent peu perçu, sauf quand un problème surgit, qui empêche cette visée ou quand un rapport poétique y prête attention. La matérialité, pour nous, n'est donc pas liée à une conception de l'espace qui en ferait une simple étendue ou un contenant, mais est bien celle par laquelle nous saisissons les opérateurs spatiaux. Ainsi, même si le travail n'est pas pleinement construit en objet géographique (Thémines et Le Guern, 2014), le fait que sa dimension spatiale apparaisse nettement, sans être thématisée cependant, chez les spécialistes du travail, nous invite à tenter de le faire. On peut donc considérer que, si les étudiants et les étudiantes d'histoire-géographie, du fait de leur formation initiale et des objets qu'ils ont à enseigner, ne sont pas insensibles à la dimension spatiale, alors celle-ci devrait être prise en charge dans leurs descriptions et leurs jugements sur les premières situations de travail. La dimension spatiale est-elle une ressource pour exercer sa réflexivité et penser son métier?

Par ailleurs, nous essayons de savoir si cette supposée sensibilité à la spatialité est mobilisée pour penser et construire les situations d'enseignement en fonction des objectifs de formation civique qui légitiment, en France, la polydiscipline scolaire appelée histoire-géographie. Nous empruntons à Hannah Arendt la notion de monde commun, défini comme l'espace public, celui de toute vie politique dont la pluralité est la condition (Arendt, 1983). Le monde commun suppose la pluralité et n'est pas un monde où chacun serait semblable aux autres. Il n'y a ainsi, au sens d'Hannah Arendt, de monde politique que s'il y a un espace proprement public, celui de l'expression de la pluralité des opinions. Si l'école n'est pas le monde, on peut toutefois penser qu'elle doit faire entrer les nouveaux venus dans ce monde commun et leur en permettre l'expérience. Or la condition de ce monde commun est l'existence d'objets fabriqués 
par les êtres humains (ce monde commun n'est pas la nature) qui peuvent séparer ou relier les personnes (ibid., p. 92). Dans une classe, les objets placés à proximité des personnes (tableaux, tables, cahiers, etc.) sont des voies d'accès à ce monde commun et des moyens d'éprouver celui-ci; ou, au contraire, ils font obstacle à cet accès: tout dépend des usages qui en sont faits. Ce croisement interdisciplinaire (géographie et philosophie) permet d'établir une lecture politique de la spatialité des situations de classe en posant la question de savoir si ces situations relèvent d'un exercice de domination ou si elles témoignent, au contraire, d'un partage de l'espace et des savoirs (Le Guern et Thémines, 2017). Les étudiantes et les étudiants qui se préparent au métier de professeur d'histoire-géographie, sont-ils sensibles à cet aspect de la spatialité dans les classes? À quelles échelles, autour de quels objets et en rapport avec quels enjeux, de façon plus ou moins intuitive ou, au contraire raisonnée, la construction d'un monde commun est-elle conçue?

Enfin, il est nécessaire, pour rendre opérationnelle l'analyse des descriptions, de se doter d'une grille de lecture des catégories conceptuelles qui manifestent la conception de la spatialité dans leurs discours. Nous distinguerons à ce sujet:

- Les surfaces supports: l'espace des relations entre éléments inscrits, projetés, affichés, etc. dans les limites visibles d'un objet. Il peut s'agir d'une feuille, d'un tableau ou d'autres objets dont la propriété plane est exploitée à des fins d'apprentissage. Le référentiel de cette spatialité est un plan (espace à deux dimensions);

- Les surfaces exposées: l'espace des relations entre les objets visibles et les acteurs présents physiquement ou par médiation langagière dans la salle de classe. Le référentiel de cette spatialité se représente mentalement en secteurs ou angles "découpés» dans la sphère perceptible, à partir d'une position d'observateur, qui peut être mobile, par déplacements et par changement de direction et de focalisation du regard;

- Les espaces interfacés : l'espace composé de façon modulaire, de relations entre scènes. Le référentiel de cette spatialité est un système de relations entre acteurs, non perceptible simultanément dans son intégralité, mais que l'on reconstitue sous la forme d'un tableau à scènes multiples ou une série de scènes observées, reliées par un fil directeur qui identifie ce que chacune doit à l'autre;

- Les espaces mobiles: l'espace des mouvements par lesquels des acteurs et des objets fabriquent de l'unité ou de la différence. Le référentiel de cette spatialité est la chora, telle qu'Augustin Berque en formule la définition, soit le tissu relationnel au sein duquel prennent place êtres et objets (Berque, 2000, p. 92-93; Retaillé, 2005). La mobilité est la qualité première mise en avant dans les descriptions d'enseignement comme dans les objets d'enseignement décrits. 
Se former pour éduquer : la spatialité comme ressource(s).

Exercices de pensée critique géographique d'étudiantes et d'étudiants

\section{MÉTHODE DE RECHERCHE : ENQUÊTE SUR UN DISPOSITIF DE PORTFOLIOS D'ÉTUDIANTES ET D'ÉTUDIANTS D'HISTOIRE-GÉOGRAPHIE ACCOMPLISSANT LEURS PREMIERS STAGES}

Le matériau exploité pour cette lecture de la spatialité mise en œuvre dans la description de leur première expérience professionnelle par des étudiantes et étudiants d'histoire-géographie est un portfolio de formation. Ce portfolio est constitué dans le cadre d'une année de formation en Master 1 (M1), qui est aussi l'année de la préparation du concours (CAPES, ou Certificat d'Aptitude au Professorat du Second degré) histoire-géographie. Du point de vue du cadrage de la formation, il a vocation à structurer le parcours de professionnalisation.

Il s'agit d'un portfolio de formation. Il ne se restreint donc pas à un compte rendu d'expérience et doit permettre de constituer pour l'avenir, en deuxième année du Master (M2) et plus tard, un ensemble de pistes de réflexion et d'action. Il est censé contribuer à la continuité du parcours de formation, du M1 au M2 pour celles et ceux qui, titulaires du CAPES, deviendront stagiaires. Ce portfolio ne comporte pas de rubriques prédéfinies, mais les contraintes d'un M1 associé à la préparation du concours, ont amené à en cadrer la composition. Dès le départ, il est annoncé que des textes ou d'autres éléments (appelés "pièces») issus de blocs de formation différents, contribueront à ce portfolio également support d'évaluation. Il rassemble ainsi, de manière obligatoire, deux pièces liées au stage réalisé chaque semestre ainsi que deux pièces liées au bloc de formation à la recherche et par celle-ci, bloc qui, dans ce parcours de master, est référé aux épreuves de concours comportant des éléments d'épistémologie des disciplines. Il ne s'agit pas uniquement de stocker les meilleures fiches de lecture, préparations de cours et préparations d'oraux. On encourage le compte rendu de cheminements, de difficultés rencontrées, de progressions ressenties dans l'approche du métier.

Pour les étudiants, le portfolio appartient à un genre nouveau. Ces étudiants ont été formés pour la plupart dans des facultés d'histoire, où l'écriture universitaire est normée par la dissertation et le mémoire de recherche, et ne comporte pas de portfolio. Les étudiants sont accompagnés dans la réalisation de leur portfolio par des séances consacrées l'une au premier semestre, l'autre au second. Il leur est demandé de rassembler des matériaux, à les constituer ensuite en collection(s), puis de problématiser une ou des questions d'entrée dans le métier à partir des points de vue ainsi rassemblés.

À l'issue du premier semestre, le portfolio comprend obligatoirement quatre pièces. Le premier stage de deux semaines, en "pratiques accompagnées», leur a permis de prendre en main leur première classe, ce dont témoignent deux de ces pièces. Souvent, des éléments annexes (préparation de cours, extraits de manuels, documents du travail dans l'établissement) ont été ajoutés. À la fin de l'année, les portfolios sont présentés lors d'une séance collective. 
Se former pour éduquer : la spatialité comme ressource(s).

Exercices de pensée critique géographique d'étudiantes et d'étudiants

Les quatre manières d'appréhender la spatialité du métier de professeur, que nous allons restituer, constituent un premier résultat. Les portfolios étudiés sont ceux de la promotion inscrite à l'École Supérieure du Professorat et de l'Éducation (ESPE) de Caen en 2013-2014, et préparant le master en même temps que le concours, soit un effectif total de dix-sept, soit sept femmes et dix hommes, de 21 à 24 ans, sauf un étudiant plus âgé, en reconversion professionnelle, âgé de 35 ans, le seul à avoir fait de la géographie lors de ses trois premières années universitaires (la licence). Six réussiront le concours d'enseignement, deux femmes et quatre hommes.

\section{DE LA PAGE AU MONDE: QUATRE MANIÈRES D'APPRÉHENDER LA SPATIALITÉ DANS L'APPRENTISSAGE DU MÉTIER DE PROFESSEUR D'HISTOIRE-GÉOGRAPHIE}

Les portfolios ont été lus et comparés en utilisant comme grille de lecture les quatre types de spatialité préalablement définis. Cette grille a été conçue de façon mixte, à partir de deux recherches empiriques différentes. Elle est en effet partiellement issue d'un travail précédent qui vise à décrire et à montrer, par contraste entre différentes situations, pour des usages du tableau, que l'espace est une dimension pertinente pour caractériser, d'un point de vue didactique, le travail des enseignants et celui des élèves (Thémines et Le Guern, 2016). Quelle que soit l'origine des catégories, leur définition s'attache à dénaturaliser "l'espace» en explicitant les référentiels (multiples) qui permettent de décrire le «faire avec l'espace» des enseignants et des élèves. L'usage de la grille nécessite de prêter attention simultanément aux contenus rapportés du stage et, par conséquent, sélectionnés et jugés intéressants par leurs auteurs; aux descriptions de situations, d'actions, de productions d'élèves ainsi qu'aux descriptions et aux jugements se rapportant d'une façon plus générale à ce qu'est effectuer le métier d'enseigner de l'histoire et de la géographie.

Les quatre manières que nous distinguons et illustrons ne constituent pas une typologie de portfolios, mais un éventail ou, si l'on préfère, un répertoire des possibles observés dans le corpus.

\section{Documenter son apprentissage professionnel par le relevé d'inscriptions d'élèves}

Une première approche de la spatialité, dans les descriptions, est celle de l'espace plan, à deux dimensions, d'écrits scolaires disciplinaires. Cette spatialité-là est présente dans tous les portfolios du corpus, avec des productions d'élèves scannées ou photographiées afin d'être stockées dans le portfolio. Cette observation en apparence anodine suppose cependant que les étudiants concernés aient éprouvé la nécessité de stocker des images de ce type. Une étude similaire, qui comparait les portfolios de professionnalisation de professeurs-stagiaires d'anglais et d'histoire-géographie, 
Se former pour éduquer : la spatialité comme ressource(s).

Exercices de pensée critique géographique d'étudiantes et d'étudiants

avait montré que ces derniers, à la différence de leurs homologues en anglais, cherchaient à documenter leur entrée dans le métier par l'exhibition de productions écrites (verbales, graphiques) d'élèves. La sensibilité à la matérialité spatiale de ces professeurs-stagiaires d'histoire-géographie contrastait fortement avec l'omniprésence du discours de leurs collègues anglicistes (Le Guern et Thémines, 2012). Par ailleurs, outre que ces «images de textes» d'élèves comportant une dimension graphique dont on connaît l'importance dans la discipline scolaire (tableaux, graphes, schémas, organigrammes) ainsi que l'efficacité heuristique pour les élèves (Doussot, 2011), elles sont elles-mêmes comprises ou travaillées graphiquement par les étudiants dans l'espace plan de l'image intégrée au portfolio.

Un exemple permet de comprendre cette spatialité à l'œuvre dans la compréhension de situations ou de productions scolaires en histoire-géographie. Albane a retenu de la phase d'observation durant le stage des productions d'élèves réalisées pendant un cours sur les Traites négrières en Atlantique et l'esclavage. Elle s'est tout particulièrement intéressée à ce que le professeur, et Albane à sa suite, appelle une «carte mentale», sorte de graphe qui a pour fonction de représenter dans l'espace de la feuille tout à la fois l'espace terrestre concerné (Europe, Afrique, Antilles) et le système d'acteurs de la Traite et de l'esclavage (Annexe 1). Si Albane entre dans la description de ce produit scolaire par l'originalité et l'efficacité perçue du moyen d'enseignement que constitue cette "carte mentale», sa lecture littérale consiste à faire de cette feuille, l'empreinte de la pensée d'un élève (portfolio d'Albane, commentaire de l'annexe 1: La carte mentale d'un élève pour suivre le chemin de la pensée) :

Je présenterai une carte mentale d'un élève montrant la façon originale d'enseigner; [...] En effet, je pense intéressant d'inculquer des méthodes de travail originales pour permettre aux élèves de trouver un mode d'apprentissage ludique. [...] Celle-ci [la carte mentale] permet de suivre le schéma de la pensée. L'élève peut alors réaliser des liens entre les éléments qui lui paraissent essentiels. En quelque sorte, la carte mentale sert à faire fonctionner la logique de l'élève et lui faire comprendre par lui-même le fonctionnement du commerce.

Si le lien de type indiciel établi par Albane entre organisation graphique de la production et cheminement d'une pensée est évidemment discutable, en revanche, il souligne son intérêt pour l'exploitation de la spatialité d'une page à des fins d'apprentissage, la carte mentale étant une «représentation subjective, à travers un langage graphique (le dessin), d'une réalité spatiale par un individu ou un groupe d'individus» (Toureille, 2016: 118).

Pour Albane, comme pour un grand nombre d'étudiants et d'étudiantes qui ont stocké des évaluations d'élèves, des organigrammes et des tableaux, et les ont commentés ou parfois retravaillés graphiquement (soulignements, encadrés et commentaires), l'écriture de l'apprentissage professionnel se focalise sur des surfaces 
Se former pour éduquer : la spatialité comme ressource(s).

Exercices de pensée critique géographique d'étudiantes et d'étudiants

supports, retenues pour la puissance d'expression de ce qui y est, selon eux, inscrit: la pensée des élèves.

\section{Faire des objets des opérateurs spatiaux d'apprentissage des élèves}

Une deuxième approche de la spatialité professionnelle est celle d'un espace, cette fois-ci à trois dimensions, associant une surface plane d'inscription ou de projection (un écran, un tableau, une affiche, un mur, etc.), les élèves et un ou des objets inscrits ou projetés (un diaporama, un schéma tracé à la main, un croquis dont la légende est remplie au fur et à mesure). Cette spatialité, à la différence de la précédente, inclut la corporéité du professeur, et, singulièrement, son placement, ainsi que celui des élèves, ce que Michel Lussault qualifie de micro-géographie comme «microchorégraphie des interactions spatiales entre les individus, et entre eux et les choses, les objets» (Lussault, 2018: 5). Elle ne déconnecte pas les savoirs enseignés de la façon de les construire, tant en direction des élèves que des dispositifs matériels. Elle est souvent caricaturée, dans le genre du compte rendu de visite de formation, sous l'expression «maîtrise de l'espace», et consiste à parler de la position (stationnement et circulation, rapprochement et éloignement dans un espace ramené à un plan). Elle est aussi rendue transparente par les catégories professionnelles et institutionnelles de la méthode et des outils. Or cette spatialité prend en compte les surfaces exposées aux regards, aux touchers, aux manipulations et aux reprises (Boutet, 2011: 39). Plusieurs étudiants ont restitué cette approche dans l'analyse de leurs premiers pas, sans toutefois se détacher pleinement du registre de la méthode.

Prenons le cas du portfolio de Francis. Celui-ci se pose d'abord des questions sur sa vision et sa pratique débutante du métier (extrait du portfolio de Francis):

Mon objectif est donc de découvrir quelle est ma méthode (sachant que chacun a la sienne, autant découvrir laquelle je souhaiterai pouvoir mettre en place), néanmoins comment se la forger en tenant compte des contraintes; interdictions; différents publics... que peut être amené à rencontrer un enseignant?

Il présente une étude de cas consacrée à la région, Une organisation dans l'Europe: la Basse-Normandie, laquelle amène les élèves à travailler l'argumentation (extrait du portfolio de Francis, commentaire de l'annexe 2. L'organigramme d'un groupe d'élèves) :

Aussi, après la réalisation d'un travail de recherche, guidé au travers d'un document TP fourni aux élèves numériquement via le réseau pédagogique, ces derniers sont amenés à réaliser (par groupe) à partir du logiciel popplet un organigramme reprenant le fil de leur argumentation afin de répondre à la problématique proposée pour l'étude de cas. 
Se former pour éduquer : la spatialité comme ressource(s).

Exercices de pensée critique géographique d'étudiantes et d'étudiants

Il aborde le thème de sa place, même s'il le fait en termes de rôle (animer, étayer) plus que de positionnement dans l'espace de travail des élèves. Cependant, en fin d'analyse, il reconnaît que (ibid.):

L'utilisation de ce genre d'outil nécessite une excellente connaissance de son utilisation par l'enseignant afin de pouvoir orienter/aider l'élève en cas de difficulté technique et tend à mettre l'enseignant dans une position plus inconfortable que le schéma classique d'un cours avec des élèves assis à leur table en train d'écouter l'enseignant.

La description signe sa prise de conscience de l'importance de la question spatiale, relativement à des intentions d'apprentissage.

L'ensemble de ces apports descriptifs et analytiques désigne la question professionnelle de l'organisation spatiale des apprentissages autour d'objets, qui peuvent ou non, selon les usages qui en sont faits, être considérés comme des opérateurs. Ici, les ordinateurs équipés de logiciels auxquels les élèves sont rapidement habitués fonctionnent comme tels. Les apprentissages s'y réalisent par une mise en réseau avec des sites Web présélectionnés et, à l'interne, par le partage des réalisations au sein de la classe. L'apprentissage a lieu là, sur ce réseau, à partir d'un espace conçu pour cela, depuis le choix du local d'enseignement, l'établissement du scénario et la délimitation des possibilités de navigation des élèves. Alors que la culture matérielle échappe souvent à la pensée des débutants, elle surgit ici comme une matérialité opératrice et non une matérialité subie, pure contrainte. La matérialité présentée dans ce portfolio de formation est une condition de possibilité de l'activité professionnelle, et signe ainsi l'exercice d'une pensée critique et puissante au sens de l'agency ou pouvoir d'agir (Clot et Simonet, 2015).

\section{De l'établissement, faire terrain: enquêter sur son environnement scolaire}

La troisième spatialité décrite dans les portfolios est celle d'espaces du travail emboîtés ou articulés les uns aux autres, à partir de la salle de classe ou en fonction de celle-ci, qui est au centre des préoccupations. Deux (2) étudiantes et 3 étudiants sur 17 explorent ainsi l'établissement par identification et mise en relation de ce qui apparaît comme compartiments à partir de l'exploration qu'ils ont conduits tout au long du stage. L'expression de cette spatialité suppose que l'étudiant se soit assuré de produire des données à partir de propos et de pratiques d'acteurs autres que luimême, son professeur d'accueil et les élèves, en particulier concernant les espaces de vie et de rencontres de ces derniers dans l'établissement. Généralement, un thème sélectionné comme fil directeur du portfolio guide la mise en relation de ces espaces, que les étudiants ont désignée comme étant leur «environnement scolaire». 
Se former pour éduquer : la spatialité comme ressource(s).

Exercices de pensée critique géographique d'étudiantes et d'étudiants

Carla est d'abord attentive à «la discipline mise en place parce que celle-ci s'effectue selon des modalités bien précises et ne correspond pas réellement à ma propre expérience du collège». Elle relate le terrain qu'elle construit à partir de la première découverte des classes de sa professeure d'accueil. "Nous avons également pris connaissance de l'environnement scolaire en passant plusieurs entretiens avec des assistants d'éducation, la documentaliste, le principal-adjoint, les professeurs, les CPE [Conseiller Principal d'Éducation] ou encore les élèves en tant que tels. Nous avons de plus passé une soirée à l'internat avec les élèves internes et suivi plusieurs classes d'études, des réunions (parents-professeurs, d'orientation) et de soutien scolaire.» Les premiers entretiens la mettent sur la piste des publics en difficulté, qui sera le fil directeur de son portfolio (extrait du portfolio de Clara) :

En fait, beaucoup de règles sont mises en place dans ce collège pour veiller à ce que tous puissent avoir leurs chances et évoluer dans un environnement scolaire le plus avantageux possible. Nous avons suivi des classes de ODP3 (option découverte professionnelle) pour justement prendre conscience des difficultés des élèves de l'établissement et comprendre quelles sont les méthodes mises en place pour lutter contre ces difficultés voire futurs échecs. Nous sommes alors allés assister à des réunions d'orientation entre professeurs et principal pour remarquer que des stages sont constamment recherchés ainsi que des débouchés professionnels. Tout ceci m’a conduite à m'intéresser à cette thématique de la discipline dans les établissements et notamment des rapports professeurs/élèves mais aussi professeurs et cadres administratifs ou encore professeurs et assistants d'éducation. Tout ceci m'amène à tenter de comprendre comment il est possible d'adapter son enseignement (et de façon plus large l'environnement scolaire) à des élèves en difficultés tout en gardant un enseignement favorisant l'ensemble des classes il s'agit véritablement de prendre en compte les difficultés, cerner les élèves, les comprendre et chercher à adapter l'enseignement selon chacun d'entre eux. Il en est de même pour l'internat, où comme je l'ai dit un peu plus haut, les élèves sont très encadrés, ils doivent notamment travailler sur certaines heures, ils peuvent obtenir de l'aide etc., mais d'autre part (et ce qui est plus étonnant), ils n'ont pas le droit de faire leurs devoirs dans leur chambre, sauf exception. Cela leur permet, selon le principal-adjoint, de créer une séparation entre d'un côté le lieu pour dormir et s'amuser et d'un autre le lieu pour travailler qui va être le CDI ou la salle de l'Internat.

La spatialité de «l'environnement scolaire» se rapproche de la catégorie des espaces interfacés: l'étudiante réalise par l'enquête l'interfaçage d'espaces et de lieux qui, pour elle, font système, de la classe qu'elle prend en main à l'établissement et aux familles. Le nombre d'acteurs présents dans la description est considérable. L'auteure qui les relie ainsi est présente, par son récit, dans les différents espaces d'établissement qui se constitue en terrain pour elle. 
Se former pour éduquer : la spatialité comme ressource(s).

Exercices de pensée critique géographique d'étudiantes et d'étudiants

\section{L'espace mobile comme référence dans l'expression de processus d'apprentissage}

Une dernière spatialité à distinguer dans les portfolios s'attache à la description de mobilités. Ces dernières peuvent être des objets d'enseignement, des outils pour l'enseignement ou encore des propriétés des auteurs qu'ils choisissent de mettre en avant. Une comparaison avec une population d'étudiants se préparant à l'enseignement d'autres disciplines serait intéressante pour cerner, par contrastes, la dimension disciplinaire de l'usage de cette spatialité. En effet, on pourrait attribuer la sollicitation de cette spatialité dans l'expression d'apprentissages professionnels, au lien établi historiquement entre mobilité ou voyage et éducation ou formation (la Bildung de la formation de soi de l'éducation classique), ou bien encore à la reprise d'un lieu commun attribuant comme finalité première à l'école de préparer les élèves au monde de demain, un monde mondialisé, tout en flux et en instabilités. Pour autant, l'insistance des étudiantes et étudiants à choisir de montrer dans les portfolios des cours qui peuvent être rattachés à ce thème, tout comme leur prédilection à prendre la métaphore du chemin pour évoquer leur apprentissage, nous semblent plaider pour un élément de l'univers disciplinaire de l'histoire-géographie.

Emblématique de cette spatialité de la mobilité, le portfolio de Barth assemble autour d'un fil directeur de type «parcours personnel» différents éléments glanés dans ses expériences antérieures ainsi que dans le stage qu'il vient de réaliser, notamment au sein d'une classe européenne (cours de géographie en anglais), avec l'enseignement en classe de terminale des dynamiques urbaines (extrait du portfolio de Barth) :

Les possibilités de combinaison et d'interaction avec d'autres enseignements sont très nombreuses: Anglais, mais aussi éventuellement selon les établissements, les autres LVE [Langues Vivantes Étrangères] ayant un enseignement d'Histoire-Géographie (par exemple un projet commun ou chacun présente son côté d'un conflit). Ces sections européennes augmentent d'autant plus l'intérêt des voyages scolaires. Il ne s'agit plus seulement de sortie liée à une seule discipline. Cela ouvre et élargit d'autant les possibilités d'échanges scolaires.

L'intérêt est aussi d'aborder des thèmes qui apparaissent peu, voire jamais, dans les cursus habituels: histoire du Royaume-Uni et des États-Unis en l'occurrence. À titre personnel, je m'intéresse particulièrement à la Guerre de Sécession et cela donnerait l'occasion de l'enseigner en classe.

Au-delà de la possibilité de travailler sur des thèmes différents, il y a aussi l'approche différente de l'enseignement des disciplines dans d'autres pays. Cela nécessiterait un gros travail personnel mais il peut être envisageable d'enseigner l'Histoire ou la Géographie telle qu'elle est enseignée en Grande-Bretagne ou aux États-Unis et ouvrir ainsi les élèves non seulement à des thématiques nouvelles, mais aussi à des méthodologies différentes. 
Se former pour éduquer : la spatialité comme ressource(s).

Exercices de pensée critique géographique d'étudiantes et d'étudiants

Dans cette spatialité, la mobilité est non seulement du déplacement, mais aussi une question d'abords ou d'approches de la nouveauté avec laquelle la mobilité permet d'établir un contact, mais aussi un processus de changement dû au fait d'être en mouvement, ainsi qu'une ressource d'apprentissage pour répondre aux finalités de l'enseignement de l'histoire-géographie.

\section{DISCUSSION}

Que nous apprennent ces portfolios d'étudiants en première année de master? À les analyser, il paraît possible et souhaitable de raisonner en termes de cohérence de formation et de compétences pour les étudiantes et étudiants. En d'autres termes, de penser ou d'instaurer des continuités plutôt que des ruptures: la géographie, en tant que discipline académique d'origine ou en tant que discipline scolaire à laquelle ils se destinent professionnellement, peut être une ressource pour eux, au-delà des contenus à enseigner. En effet, les savoirs disciplinaires contribuent à l'intelligibilité des situations professionnelles, dans et hors la classe ou, du moins, rendent sensibles à des variations d'échelle, des matérialités, des flux et des mobilités.

Toutefois, dans le corpus des portfolios analysés, le souci de la fabrique du monde commun n'apparaît à proprement parler qu'à partir du moment où l'espace n'est pas seulement celui du plan. Les travaux d'élèves scannés apparaissent comme une mosaïque de copies, toutes assignées strictement à un auteur-scripteur individuel. Ce qui n'apparaît pas alors est le lien entre les individus dans la classe, y compris avec l'enseignant. La classe est composée d'individus monades qui ne font pas vraiment société.

Cet ensemble de monades dans la classe ne fait pas «monde». C'est à partir des considérations sur les mises en relation des individus et des objets, matériels ou intellectuels, qu'un monde apparaît comme commun, partagé par des individus vus comme singuliers. Ce qui est alors fabriqué, ce sont des collectifs, ceux des élèves au sein de ce qui est appelé «la classe» (Francis) et aussi hors de la classe, dans l'établissement où se construisent également des collectifs d'adultes (professeurs, autres professionnels, parents) (Carla), ou encore au-delà de l'établissement dans une perspective d'ouverture au monde (Barth). Rendus intellectuellement visibles, ces collectifs peuvent être sources d'interrogation et le caractère nécessairement pluriel, fait de divergences, de différences et de discussions, ce qui est au sens d'Arendt la vie politique, celle où les citoyens peuvent être animés par une visée et une œuvre commune, suscite question et débat entre eux. Les productions numériques des élèves rassemblées grâce au réseau informatique local seront partagées et discutées (Francis). Les articulations que Carla souligne entre pratiques de classe et politique d'établissement installent un principe général qui se veut au fondement de l'ensemble des rapports entre acteurs dans le collège, celui de respect. Il resterait à savoir si ce principe même 
ou certains aspects de sa mise en œuvre sont effectivement mis en discussion, ce que son portfolio ne permet pas de savoir.

\section{CONCLUSION}

Au-delà du groupe disciplinaire des étudiants d'histoire-géographie, ce que nous permettent de comprendre ces portfolios est la question de savoir quelles sont les conditions d'un exercice effectif d'une pensée critique. Autrement dit, la spatialité du travail peut certainement être considérée comme un point d'attention à faire partager à tout futur professionnel et à toute future professionnelle de l'enseignement et de l'éducation. Même si cette dimension spatiale n'a pas encore pleinement été construite en objet de recherche par les analystes du travail, elle peut être documentée à partir de dispositifs tels que celui que nous proposons (portfolios constitués dans des formations universitaires disciplinaires et professionnelles par alternance). L'enjeu de formation de tels dispositifs est de rendre palpable l'insuffisance de catégories telles que: méthode, outil d'enseignement, ainsi que la faiblesse analytique et praxéologique des disjonctions entre pédagogie et didactique, compétences disciplinaires et compétences générales. L'espace, dans le travail enseignant, pourvu qu'on le conçoive comme relationnel et comme relatif aux intentions et aux actions des protagonistes, est un levier de compréhension des processus de construction ou d'exclusion des apprentissages. C'est en ce sens que nous affirmons que la géographie, en formation professionnelle, ça sert d'abord à faire un monde commun. Idéalement, la géographie académique devrait pouvoir être un outil puissant de formation professionnelle, pour tous les enseignants et plus largement pour tous les métiers de l'encadrement éducatif, une «majeure» (Lussault, 2018, p. 5) de la formation, surtout quand il s'agit de former pour éduquer.

\section{Références bibliographiques}

ARENDT, H. (1983). Condition de l'homme moderne. Paris: Calmann-Lévy [The Human Condition, 1961, trad. G. Fradier].

BERQUE, A. (2000). Ecoumène. Introduction à l'étude des milieux humains. Paris: Belin.

BOUTET, J. (2011). En regard: les écrits professionnels des enseignants, traces ou pratiques. Dans B. Daunay (dir.). Les écrits professionnels des enseignants. Approche didactique (p. 35-41). Rennes: Presses Universitaires. 
CLOT, Y. et SIMONET, P. (2015). Pouvoir d'agir et marges de manœuvre. Le travail humain, 78, 31-52. Repéré à https://www.cairn.info/revue-le-travail-humain-20151-page-31.htm

DOUSSOT, S. (2011). Didactique de l'histoire. Outils et pratiques de l'enquête historienne en classe. Rennes: Presses Universitaires.

LE GUERN, A.-L. et THÉMINES, J.-F. (2017). Monde en partage ou espace de domination: objets ordinaires dans les salles de classe. Dans Y. Bonny, N. Bautès et V. Gouëset (dir.), L’espace en partage. Approche interdisciplinaire de la dimension spatiale des rapports sociaux (p. 253-271). Rennes: Presses universitaires.

LE GUERN, A.-L. et THÉMINES, J.-F., (2012). Discipline et rapport au monde professionnel: le portfolio comme self assessment. Pratiques. Repéré à https:// journals.openedition.org/pratiques/1947

LUSSAULT, M. (2018). Mettre l'expérience extrascolaire en lien avec la pratique scolaire. Entretien avec Régis Guyon. Diversité. Revue d'actualité et de réflexion pour l'action éducative, 191. Répéré à https://cdn.reseau-canope.fr/archivage/ valid/contenus-associes-entretien-avec-michel-lussault-N-15271-22828.pdf

LUSSAULT, M. (2010). Ce que la géographie fait au(x) monde(s). Tracés. Revue de sciences humaines, 10 (Hors-série À quoi servent les sciences humaines?). Repéré à http://journals.openedition.org/traces/4854

PROST, A. (1998). Un couple scolaire. Espaces/Temps: Les Cahiers, 66-67. Repéré à https://www.persee.fr/doc/espat_0339-3267_1998_num_66_1_4038

RETAILlÉ, D. (2005). L'espace mobile. Dans B. Antheaume et F. Giraut (dir.). Le territoire est mort, vive le territoire! Une (re) fabrication au nom du développement (p. 175-201) Paris: IRD Éditions.

THÉMINES, J.-F. (2006). Le rapport pratique à l'épistémologie, chez les professeursstagiaires du secondaire en géographie. Cybergeo: European Journal of Geography. Répéré à https://journals.openedition.org/cybergeo/2490

THÉMINES, J.-F. et LE GUERN, A.-L. (2016). Les apprentissages dans la classe: décrire l'espace à partir de photographies. Dans C. Cohen-Azria, M.-P. Chopin et D. Ravachol Orange (dir.), Les méthodes de recherche en didactiques: questionner l'espace (p. 35-49). Villeneuve d'Asq: Presses Universitaires du Septentrion.

THÉMINES, J.-F. et LE GUERN, A.-L., (2014). Construire en objet géographique l'entre-deux du travail ordinaire. Carnets de géographes, 3. Répéré à https:// journals.openedition.org/cdg/446 
TOUREILLE, E. (2016). Cartes mentales et autres techniques projectives visuelles. Dans M. Morange et C. Smoll (dir.), Les outils qualitatifs en géographie. Méthodes et application (p. 117-139). Paris: Armand Colin.

\section{Annexe 1}

\section{La carte mentale d'un élève pour "suivre le schéma de la pensée" (extrait du portfolio d'Albane)}

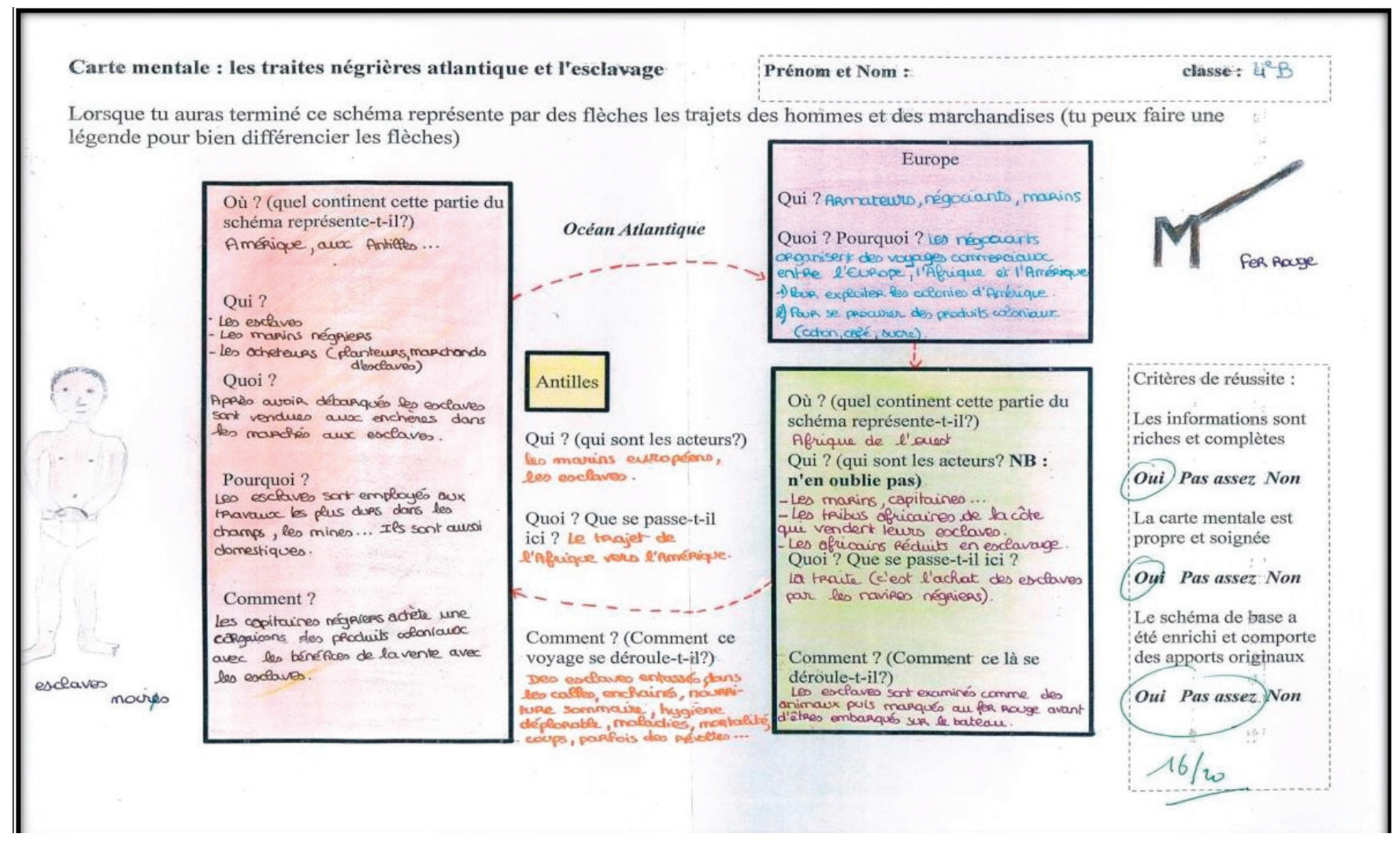




\section{Annexe 2}

L'organigramme d'un groupe d'élèves sur logiciel Popplet (extrait du portfolio de Francis)

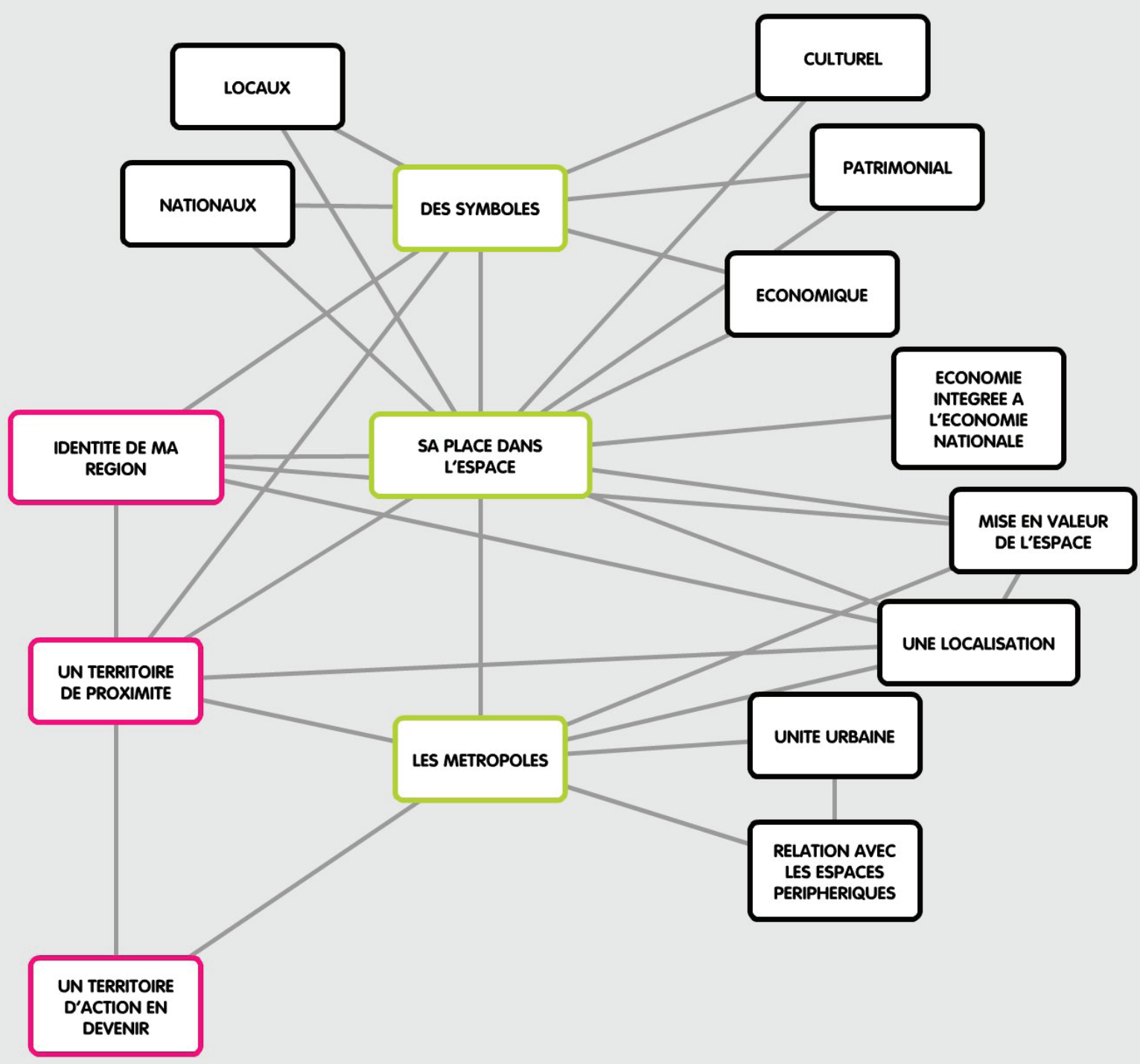

